\title{
Global characteristics of the correlation and time lag between solar and ionospheric parameters in the 27-day period
}

\author{
Choon-Ki Lee ${ }^{\mathrm{a}, *}$, Shin-Chan Han ${ }^{\mathrm{b}, \mathrm{c}}$, Dieter Bilitza ${ }^{\mathrm{d}, \mathrm{e}}$, Ki-Weon Seo ${ }^{\mathrm{a}}$ \\ ${ }^{a}$ Division of Polar Earth-System Sciences, Korea Polar Research Institute, 12 Gaetbeol-ro, Yeonsu-gu, Incheon 406-840, Korea \\ b Planetary Geodynamics Laboratory, Code 698, NASA Goddard Space Flight Center, Greenbelt, MD 20771, USA \\ ' University of Maryland at Baltimore County, 1000 Hilltop Circle, Baltimore, MD 21250, USA \\ ${ }^{\mathrm{d}}$ Heliophysics Laboratory, Code 672, NASA Goddard Space Flight Center, Greenbelt, MD 20771, USA \\ e Space Weather Laboratory, George Mason University, 4400 University Drive, Fairfax, VA 22030, USA
}

\section{A R T I C L E I N F O}

\section{Article history:}

Received 24 October 2011

Received in revised form

19 January 2012

Accepted 22 January 2012

Available online 2 February 2012

Keywords:

Ionosphere

Electron density

GRACE

CHAMP

\begin{abstract}
A B S T R A C T
The 27-day variations of topside ionosphere are investigated using the in situ electron density measurements from the CHAMP planar Langmuir probe and GRACE K-band ranging system. As the two satellite systems orbit at the altitudes of $\sim 370 \mathrm{~km}$ and $\sim 480 \mathrm{~km}$, respectively, the satellite data sets are greatly valuable for examining the electron density variations in the vicinity of F2-peak. In a 27-day period, the electron density measurements from the satellites are in good agreements with the solar flux, except during the solar minimum period. The time delays are mostly 1-2 day and represent the hemispherical asymmetry. The globally-estimated spatial patterns of the correlation between solar flux and in situ satellite measurements show poor correlations in the (magnetic) equatorial region, which are not found from the ground measurements of vertically-integrated electron content. We suggest that the most plausible cause for the poor correlation is the vertical movement of ionization due to atmospheric dynamic process that is not controlled by the solar extreme ultraviolet radiation.
\end{abstract}

(c) 2012 Elsevier Ltd. All rights reserved.

\section{Introduction}

The Earth's ionosphere is strongly controlled by solar extreme ultraviolet irradiance. It is therefore not surprising that the 27-day periodicity observed in solar flux recordings, that is due to the solar rotation period, can also be observed in ionospheric parameter recordings like the $F$ peak plasma frequency, foF2, and the total ionospheric electron content, TEC. Nevertheless, some earlier studies have found only a weak correlation between these parameter's 27-day periodicities (Doherty et al., 2000; Richards, 2001). It was suggested that, besides the solar extreme ultraviolet (EUV) forcing, other factors could affect the local ionosphere parameters and at times even more strongly than the solar EUV flux. They include neutral winds, plasma vertical drift, vertical coupling in the atmosphere-ionosphere system through atmospheric waves (tidal and planetary waves), and geomagnetic activity (Rich et al., 2003).

Unlike the local ionospheric parameters, the global mean TEC measured by GPS and as compiled in the Global Ionospheric Map (GIM) shows a correlation with the solar activity proxy such as the radio flux at $10.7 \mathrm{~cm}$ (F10.7) or the Mg II index (Afraimovich

\footnotetext{
* Corresponding author. Tel.: +821036359798.

E-mail address: cklee92@kopri.re.kr (C.-K. Lee).
}

et al., 2008; Hocke, 2008). The Defense Meteorological Satellite Program (DMSP) satellite also observed a 27-day variation in plasma density with an amplitude of up to $40-50 \%$ at the altitude of $\sim 840 \mathrm{~km}$ (Rich et al., 2003). A similar result was found from the analysis of KOMPSAT- 1 measurements at $\sim 675 \mathrm{~km}$ (Min et al., 2009).

In this study, we investigate the 27-day ionospheric modulation at the altitudes of $\sim 370 \mathrm{~km}$ and $\sim 480 \mathrm{~km}$ close to the F2 peak by analyzing the electron density measured by the CHAllenging Minisatellite Payload (CHAMP) planar Langmuir probe (PLP) and the Gravity Recovery and Climate Experiment (GRACE) K-band ranging system. We study the spatial dependence of cross-correlations between the electron density measurements and the solar flux measurements globally. We also discuss the altitude dependence of the 27-day ionospheric variations by analyzing the in situ satellite measurement and the ground-based TEC from GPS.

\section{Data and method}

The CHAMP satellite measures the electron density with the on-board PLP instrument every 15 seconds in a near-circular orbit with an inclination of $87.3^{\circ}$ (Reigber et al., 2002). Its mean altitude decreased from $\sim 450 \mathrm{~km}$ in 2000 to $\sim 320 \mathrm{~km}$ in 2009 . 
The orbital configuration is such that CHAMP sweeps through all local times every 131 day allowing the satellite to monitor the global ionospheric climatology. The two GRACE satellites measure inter-satellite distance changes every 5 seconds from the dualfrequency K-band ranging system at an orbit altitude of $\sim 480 \mathrm{~km}$ and an inclination of $89^{\circ}$ (Tapley et al., 2004). The phase difference between the $\mathrm{K}$ - and Ka-band signals can be used to deduce the (biased) electron content between the two GRACE satellites. The electron density at the intermediate position between the two satellites is approximated by the electron content divided by the baseline length of $\sim 220 \mathrm{~km}$ (Lee et al., 2011).

We use two solar flux measurements, F10.7 index and SOHO/ SEM, to derive the correlation with the electron density in ionosphere. The F10.7 indicates the solar radiation flux on the wavelength of $10.7 \mathrm{~cm}$ in s.f.u. units $\left(10^{-22} \mathrm{Wm}^{-2} \mathrm{~Hz}^{-1}\right)$ (Tapping and Charrois, 1994), and has been most widely used for ionospheric studies and monitored over 60 years. Recently, the solar EUV radiation in two bands $(0.1-50 \mathrm{~nm}$ and $26-34 \mathrm{~nm}$ ) has been measured by the Solar EUV Monitor (SEM) spectrometer aboard the Solar Heliospheric Observatory (SOHO) (Judge et al., 1998). We use the SOHO/SEM daily averages in the $0.1-50 \mathrm{~nm}$ band since the two bands are strongly correlated. Although it is apparently nonlinear with F10.7, the solar EUV radiation from SOHO/SEM is more linearly correlated with a solar activity factor P, (F10.7+F10.7A)/2 (Liu et al., 2006). Thus a few extremely large or small records in SOHO/SEM apart from the linear relation with $P$ are removed and then interpolated using their neighbors.

The 27-day periodic variation in the satellite measurements is modulated by long-period trends. As discussed in Rich et al. (2003) and Oinats et al. (2008), the percentage change is more useful than the original time-series of measurements for computing the cross-correlation with the solar flux recordings. We take the following steps to compute the cross-correlation of the percentage change: (i) All electron density measurements within a day are averaged (denoted by $N_{d}$ ) to be comparable to the daily F10.7; (ii) By using a finite impulse response low-pass filter, we remove short-period ( $<13$ day) variations, such as the strong periodicities of 7 and 9 day that might be associated with variations in solar wind high-speed streams and geomagnetic activity and that are generally not observed in the F10.7 data (Thayer et al., 2008); (iii) Long-period trends are removed by subtracting the 27-day running average $\bar{N}_{d}$ from the low-pass filtered daily electron density $\hat{N}_{d}$ and then a percentage change $\delta N_{d}$ is calculated by $\delta N_{d}=\left(\hat{N}_{d}-\bar{N}_{d}\right) / \bar{N}_{d}$; (iv) The percentage changes of the F10.7 and the SOHO/SEM are also calculated following the same steps (ii) and (iii); (v) The time-series of electron density measurements and SOHO/SEM have an effective time tag of $12 \mathrm{~h}$ UT because we have averaged the data over a day; conversely, the F10.7 has a time tag of $20 \mathrm{~h}$ UT since it is measured at noon (local time) at Penticton, Canada (Tapping and Charrois, 1994). In order to match the time tags of F10.7 and others and improve the resolution of time delay, all time-series are resampled every $8 \mathrm{~h}$ at time tags of $4 \mathrm{~h}, 12 \mathrm{~h}$, and $20 \mathrm{~h}$ UT by a cubic spline interpolation; (vi) Finally, the correlation functions between the two time-series of percentage changes in the electron density and the solar flux are calculated with a time lag varying from -5 to 5 day. The correlation coefficient $(C)$ and time delay (D) are defined as the maximum value of the correlation function and the corresponding time lag, respectively (Oinats et al., 2008).

The ground-based TEC data in our study are from the CODE (Center for Orbit Determination in Europe) GIMs (http://aiuws. unibe.ch/ionosphere). The GIM TEC (CHAMP) and GIM TEC (GRACE) represent the TEC interpolated at the time and location of the CHAMP and GRACE measurements, respectively.

\section{Results}

The time-series of daily-averaged electron densities $\left(N_{d}\right)$ from CHAMP and GRACE are characterized by annual, semiannual, and 27-day variations with a decreasing trend due to the 11-year solar cycle (Fig. 1). Our low and high-pass filtering removes most of these variations and the percentage change shown in the lower part of the panels in Fig. 1 is dominated by the 27-day variation. The percentage change of solar flux is visibly correlated with the percentage change of the satellite measurements. To study the time-dependence of the correlation, we divide the time-series into the overlapped subsets and then calculate the correlation coefficient in each subset. The length of subset segments is 81 day and the interval between two consecutive subsets is 27 day. For an 81-day sample length, the correlation coefficient corresponding to the 0.01 significance level (99\% confidence level) is 0.285 in Strudent's t distribution. In general, the correlation varies from 0.7 to 0.9 depending on time, as shown in Fig. 2. The correlation coefficient is decreasing with decreasing solar activity because with diminishing solar influence other effects like the meteorological forcing from below become more influential. The magnitude of the meteorological forcing does not depend on solar activity and therefore during low solar activity it is responsible for a larger percentage of the ionospheric variability than during high solar activity. Another possible cause of the de-correlation with diminishing solar influence is the random noise in the solar flux measurements because, for example, the F10.7 variation at the solar minimum is only in the range of \pm 0.7 S.F.U. close to the accuracy of F10.7 index. The SOHO/SEM is more highly correlated with the electron densities than the F10.7 is $(C=0.84$ for F10.7CHAMP, $C=0.85$ for F10.7-GRACE, $C=0.90$ for SOHO/SEM-CHAMP, and $C=0.89$ for SOHO/SEM-GRACE, averaged from 2003 to 2007),
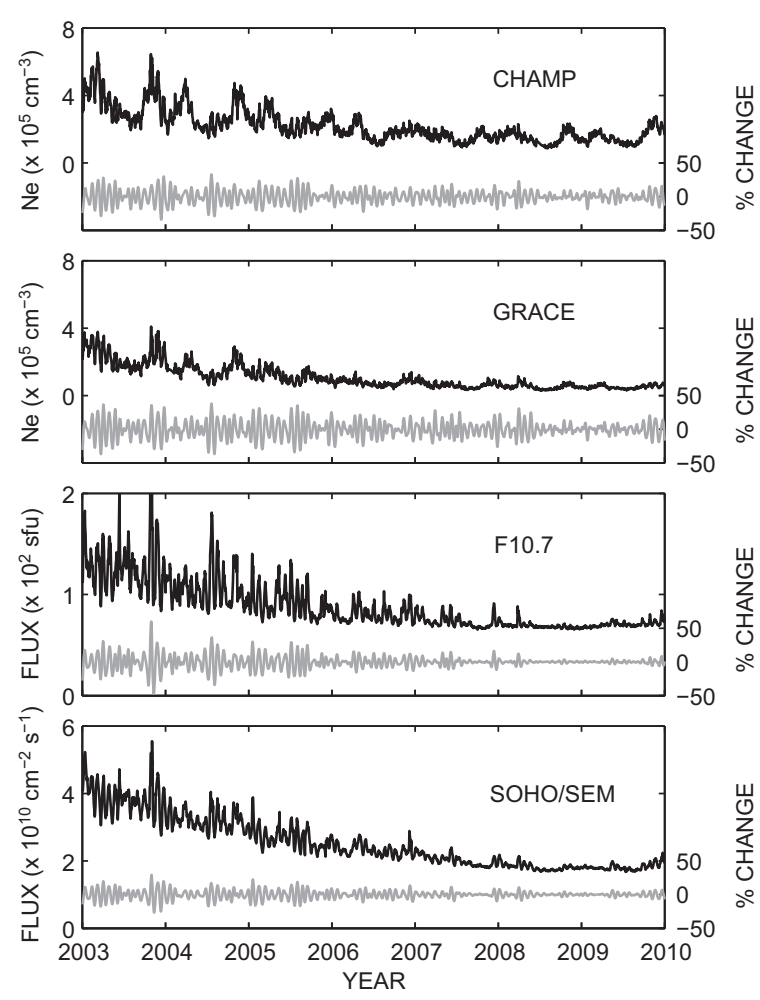

Fig. 1. Time-series of the daily averaged electron density from CHAMP and GRACE and of the F10.7 index and the solar EUV radiation in the $0.1-50 \mathrm{~nm}$ wavelength (SOHO/SEM). They are depicted in black. The percentage changes of the daily values are shown in gray. 
a
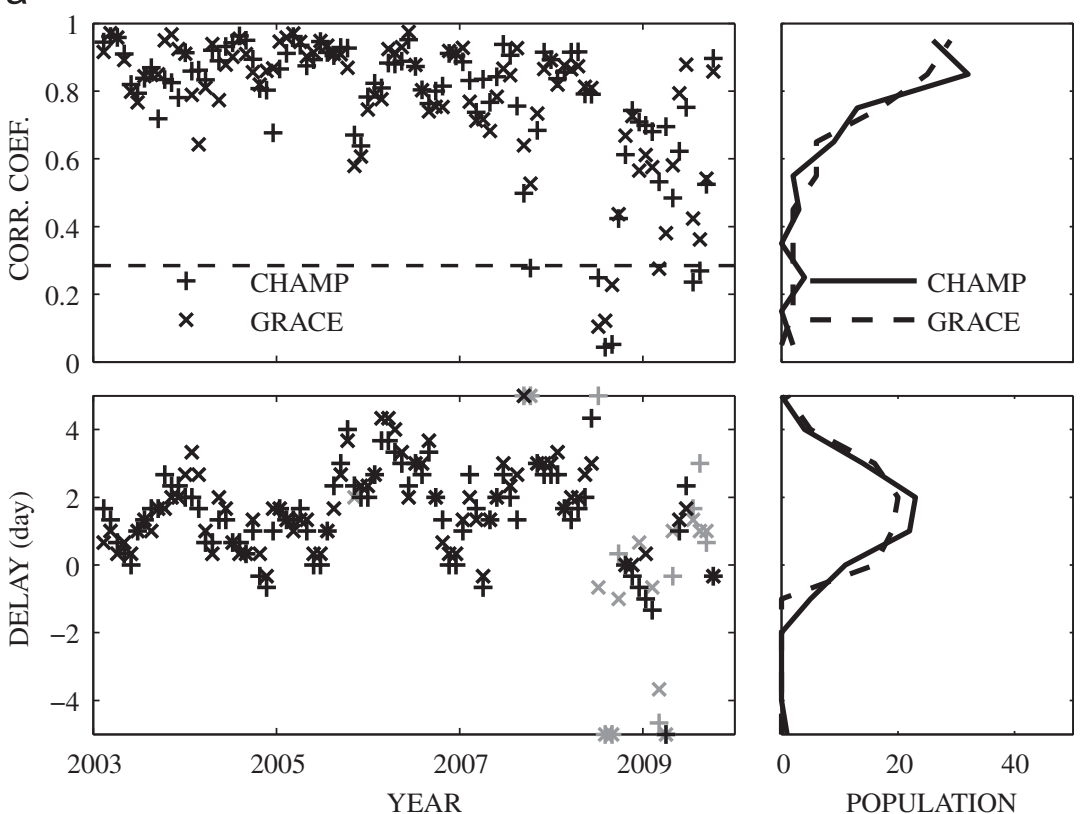

b
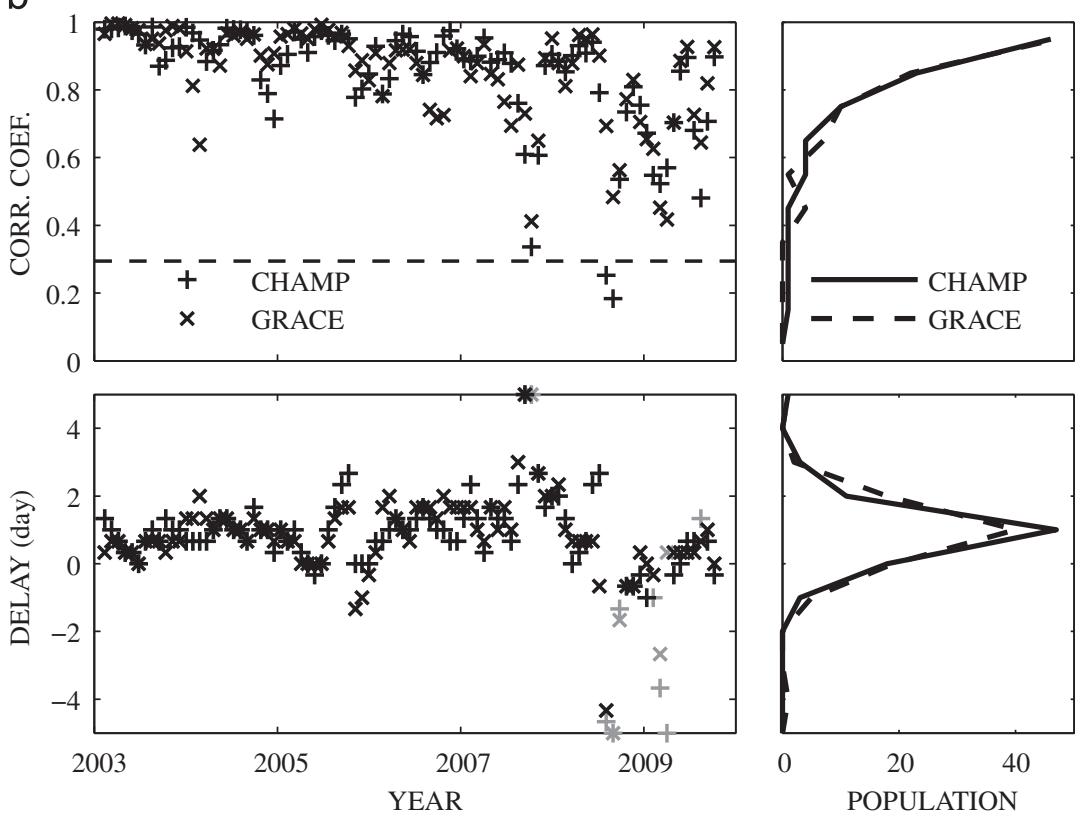

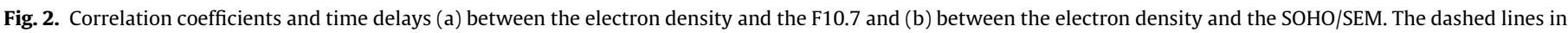

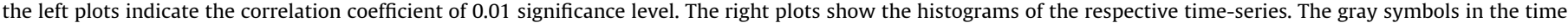
delay are only from the sub-set data with low correlations ( 0.6 or below), which are excluded for the histogram.

representing that the SOHO/SEM is a better proxy for ionospheric parameters than F10.7.

The temporal distribution of the time delay is also shown in Fig. 2. The electron density dominantly correlates best with the solar flux at a time lag of 1-2 day. This means that it takes 1-2 day for changes in solar activity to document themselves in the ionosphere. Similar time lags have been reported based on radio beacon and GPS-TEC observations (Jakowski et al., 1991, 2002). Such a time lag may be caused by the long lifetime of atomic oxygen, a major source for ionospheric electrons and $\mathrm{O}^{+}$ions. $\mathrm{O}$ is created at lower altitudes $(\sim 100 \mathrm{~km})$ by photo-dissociation, and then diffuses upward where neutral densities are much lower and chances for the required 3-body collisions are very small resulting in $\mathrm{O}^{+}$lifetimes of one to several days. From 2003 to 2007, the time delay for F10.7 ( $1.7 \pm 1.2$ day) is larger than that for SOHO/ SEM (1.1 \pm 1.0 day), as locally observed (Maruyama, 2010). Large delays ( $>3$ day) are observed from F10.7 in year 2004 and every 2 years, which are not found in the SOHO/SEM. They induce such averaged difference in the time delay estimates ( $\sim 0.6$ day).

The spatial patterns of correlation and time delay are computed by locally averaging the electron density measurements in the period from 2003 to 2007. In a specific area, the ascending and descending track represent the observations with a 12-hour local time difference. Therefore, we grouped the satellite measurements into ascending and descending track sets and then calculate the correlation and time delay. In each set, the data within a day are binned and averaged on an $18 \times 16$ grid (i.e., $10^{\circ}$ in latitude and $22.5^{\circ}$ in longitude) that corresponds to the satellites' 

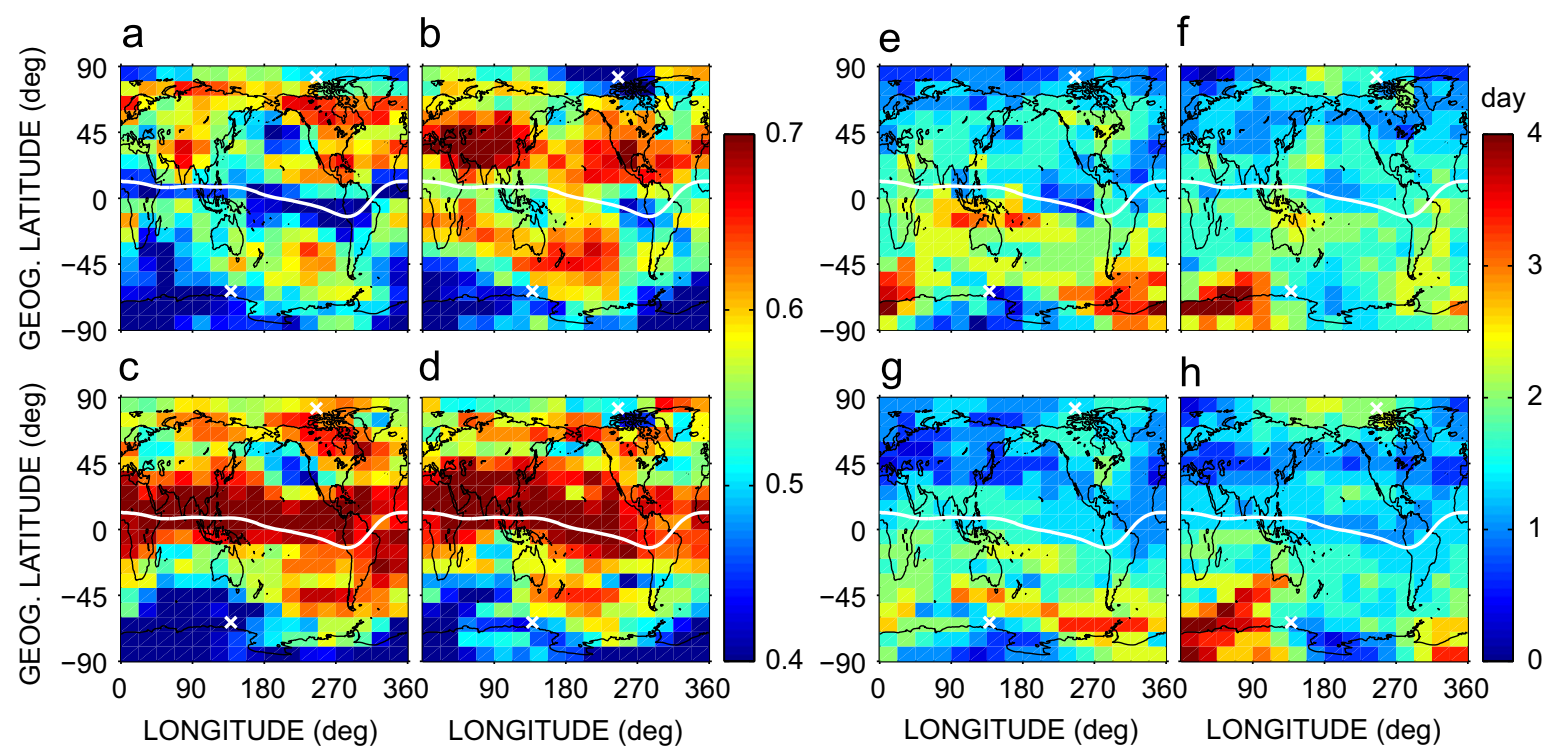

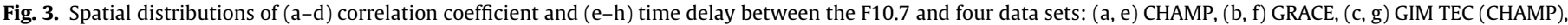
and $(\mathrm{d}, \mathrm{h})$ GIM TEC (GRACE). The white line and white cross indicate the magnetic equator and the magnetic pole, respectively.

sampling interval of 16 revolutions per day. The time-series of averaged electron density in each grid are processed following the procedure described in the previous section. No significant difference is found between the results from ascending and descending track sets, which allows us to look into only one set, for example, ascending track data. The time-series in each grid cell may include the local time variation as well as the seasonal variation, since the CHAMP and GRACE sweep all local time every 131 day and 160 day, respectively. However, significant changes in the correlation with respect to either local time or season are not observed.

The spatial patterns of the correlation between F10.7 and electron density on a $10^{\circ}$ (latitude) $\times 22.5^{\circ}$ (longitude) grid are shown in Fig. 3 (The ones for SOHO/SEM are similar and not shown). The highest correlation is found at mid-latitudes. The correlation coefficients are relatively low in high-latitudes and generally higher in the northern hemisphere than in the southern hemisphere. In the southern hemisphere, the low correlation extends from the South Pole to the mid-latitude over the Indian and Atlantic sectors. At high latitudes, the direct impact of the solar wind through open magnetic field lines and the downpour of precipitating particles at auroral latitudes compete with the solar EUV influence on the ionosphere. Most interestingly, at low latitudes around the magnetic equator the in situ satellite data show a low correlation while the TEC data exhibit a high correlation. At low latitudes, the atmospheric dynamic processes such as the equatorial ionization fountain (often called Equatorial Ionization Anomaly, EIA) are the dominant forces shaping the ionosphere plasma (Stening, 1992). Those distinct effects at the high and low latitudes may be responsible for the spatial pattern in the correlation of the electron density with the solar flux.

When the correlation maps from in situ satellite data near the F2-peak altitudes and the TEC from GIM are compared, we find systematic differences, particularly along the magnetic equator. Within the latitude band of $10^{\circ}$ from the magnetic equator, the correlation coefficients from in situ satellite data are low, i.e. less than 0.5 for CHAMP, whereas those of GIM TEC (CHAMP) are 0.7 or higher. A similar pattern is found for the GRACE in situ and TEC data as well, although to a lesser extent. Rich et al. (2003), on the other hand, using the DMSP satellite data, found that the correlation coefficient in the equatorial region is higher than in the mid-latitude region, similar to our results with the GIM TEC data. Recalling the altitudes of these satellites (i.e., $\sim 370 \mathrm{~km}$ for CHAMP, $\sim 480 \mathrm{~km}$ for GRACE, and $\sim 840 \mathrm{~km}$ for DMSP), our analysis suggests that the lower correlation in the equatorial region may be confined to the F2 peak altitude and its vicinity.

For the time delay, all four data sets (Fig. 3e-h) show a consistent spatial distribution. The dominant pattern is a hemispheric asymmetry with a time delay of predominantly one day in the northern hemisphere and two days or longer in the southern hemisphere.

\section{Discussion}

Studying the correlation between electron density and solar flux, we find that while many of the global correlation features are similar for F-region in situ data and corresponding GPS-TEC data there is a distinct difference in correlation behavior at and near the magnetic equator. TEC data are well correlated with solar flux by means of the 27-day solar rotation, the GRACE data, however, exhibit only a marginal correlation with solar flux, and the CHAMP data not at all. Fig. 4 shows the variations of correlation coefficients and time delays along geomagnetic latitude (for a $2^{\circ}$ resolution). A substantially decreased correlation coefficient is found around the magnetic equator $\left( \pm 30^{\circ}\right)$ for the CHAMP satellite data. Across all four data sets and both solar fluxes, the hemispherical asymmetry of time delay is apparent. The time delay in the southern hemisphere (especially $10^{\circ} \mathrm{S}-70^{\circ} \mathrm{S}$ ) is a day longer than in the northern hemisphere.

Fig. 5a indicates the altitude ranges of CHAMP and GRACE from 2003 to 2007 as well as the samples of the F2-peak heights (hmF2) predicted by the IRI-2007 model (Bilitza and Reinisch, 2008) for the time and location of the CHAMP data. The CHAMP orbits are closer to the hmF2 than the GRACE orbits and are sometimes even below hmF2 in the equatorial region. The map of mean distance from the height of CHAMP satellite to the hmF2, depicted in Fig. 5b, shows a pattern similar to the correlation map in Fig. 3a. The distance is smallest in the equatorial region and the distances over the American and Eurasian continents are larger than those over the Pacific Ocean in the northern hemisphere. These coherent spatial patterns support the idea that the decreased correlation along the magnetic equator is likely due to the proximity of the satellite orbit to the F2 peak. 

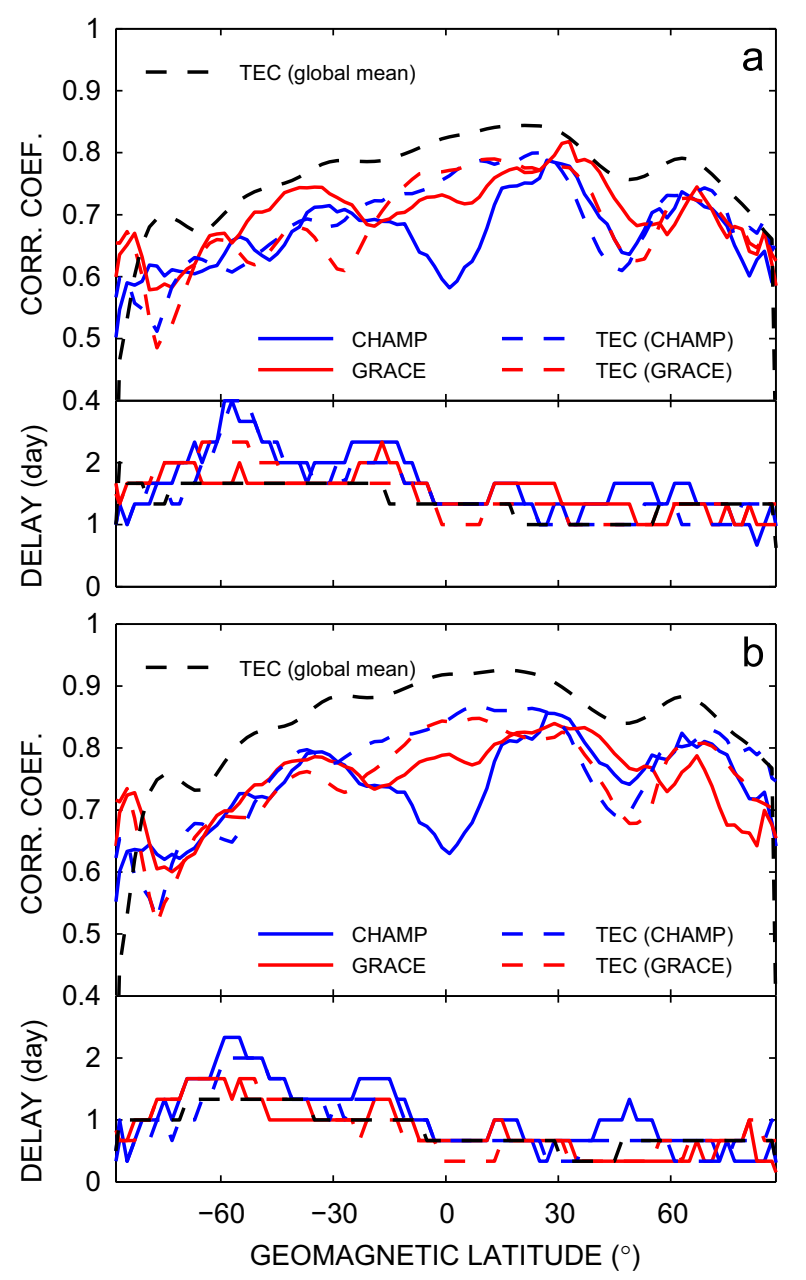

Fig. 4. The correlation coefficient and time delay between four data sets (two in situ measurements from CHAMP PLP and GRACE KBR and two TECs along CHAMP and GRACE satellite orbits) and the solar fluxes, (a) F10.7 and (b) SOHO/ SEM, along geomagnetic latitude. The black dashed lines indicate the correlation coefficients between the global mean TEC and the solar fluxes.

So what is disturbing the correlation between the electron density and the solar EUV radiation in the equatorial F2 layer? The (vertically-integrated) TEC maintains a strong correlation in the equatorial region unlike the satellite measurements near the F-region, even though the F-region ionization dominantly contributes to the TEC. This fact indicates that the ionosphere as a whole is less affected by the re-distribution of ionization causing the low correlation near the F2-peak. These distinct behaviors of electron contents near the F2-peak and in the whole ionosphere suggest that the vertical movement of ionization (e.g. ion drift constituting the EIA) near the F2-peak plays a leading role in the short-term variation of electron density near the F2-peak.

\section{Conclusion}

We have studied the correlation and time delay between the 27-day solar rotation period as seen in the solar flux and as documented in in situ electron density measurements by the CHAMP and GRACE satellites and TEC measurements from GPS. We find good correlation except at high latitudes where particle precipitation competes with the solar influence. At low latitudes the correlation also breaks down for electron density data close to the $\mathrm{F}$ peak because of the dynamic process that is not under direct solar control. As expected, we find that the correlation decreases
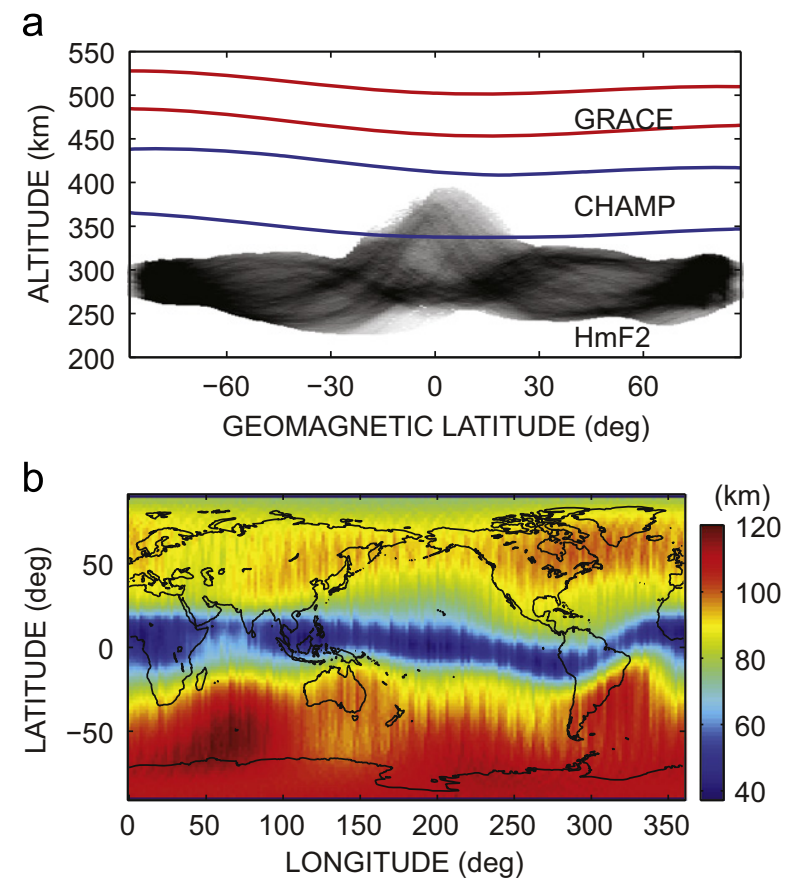

Fig. 5. (a) Sample density of the hmF2 values from IRI-2007 model, corresponding to the CHAMP data. The blue and red lines indicate the altitude range of CHAMP and GRACE, respectively. (b) Average distance from the CHAMP height and the hmF2.

towards the solar cycle minimum because of the increasing importance of influences other than solar, e.g. meteorological forcing from below. The time delay between the solar rotation as manifested in solar flux and the satellite data shows a consistent behavior across all four data sets. We find a predominantly 1-day difference of time delay between the northern and southern hemisphere. Further study is needed to investigate the causes of this hemispherical asymmetry.

The results of this study are important for an improved representation of the solar dependence in data-based ionospheric models like the International Reference Ionosphere (IRI). IRI is widely used for many space weather applications but currently only use solar dependence at a 12-month average level. With the results of this study it might be possible to introduce solar dependence at a daily F10.7 level keeping in mind the global differences in correlation and time delay.

\section{Acknowledgments}

This work was supported by NASA Earth Surface and Interior program and GRACE projects and Korea Polar Research Institute (KOPRI) projects (PE11070). We thank DLR for providing the GRACE telemetry data and JPL for producing the high-quality Level-1B products.

\section{References}

Afraimovich, E.L., Astafyeva, E.I., Oinats, A.V., Yasukevich, Y.V., Zhivetiev, I.V., 2008 Global electron content: a new conception to track solar activity. Annales Geophysicae 26, 335-344.

Bilitza, D., Reinisch, B.W., 2008. International Reference Ionosphere 2007: Improvements and new parameters. Advances in space research : the official journal of the Committee on Space Research (COSPAR) 42, 599-609.

Doherty, P.H., Klobuchar, J.A., Kunches, J.M., 2000. Eye on the Ionosphere: The Correlation between Solar $10.7 \mathrm{~cm}$ Radio Flux and Ionospheric Range Delay. GPS Solutions 3, 75-79. 
Hocke, K., 2008. Oscillations of global mean TEC. Journal of Geophysical Research 113, A04302.

Jakowski, N., Fichtelmann, B., Jungstand, A., 1991. Solar activity control of ionospheric and thermospheric processes. Journal of Atmospheric and Terrestrial Physics 53, 1125-1130.

Jakowski, N., Heise, S., Wehrenpfennig, A., Schlter, S., Reimer, R., 2002. GPS/ GLONASS-based TEC measurements as a contributor for space weather forecast. Journal of Atmospheric and Solar Terrestrial Physics 64, 729-735.

Judge, D.L., McMullin, D.R., Ogawa, H.S., Hovestadt, D., Klecker, B., Hilchenbach, M. Möbius, E., Canfield, L.R., Vest, R.E., Watts, R., Tarrio, C., Kühne, M., Wurz, P., 1998. First Solar EUV Irradiances Obtained from SOHO by the Celias/Sem. Solar Physics 177, 161-173.

Lee, C.-K., Han, S.-C., Bilitza, D., Chung, J.-K., 2011. Validation of international reference ionosphere modes using in situ measurements from GRACE K-band ranging system and CHAMP planar Langmuir probe. Journal of Geodesy 85, 921-929.

Liu, L., Wan, W., Ning, B., Pirog, O.M., Kurkin, V.I., 2006. Solar activity variations of the ionospheric peak electron density. Journal of Geophysical Research 111, A08304.

Maruyama, T., 2010. Solar proxies pertaining to empirical ionospheric total electron content models. Journal of Geophysical Research 115, A04306.

Min, K., Park, J., Kim, H., Kim, V., Kil, H., Lee, J., Rentz, S., Lhr, H., Paxton, L., 2009. The 27-day modulation of the low-latitude ionosphere during a solar maximum. Journal of Geophysical Research 114, A04317.
Oinats, A.V., Ratovsky, K.G., Kotovich, G.V., 2008. Influence of the 27-day solar flux variations on the ionosphere parameters measured at Irkutsk in 2003-2005. Advances in Space Research : The Official Journal of the Committee on Space Research (COSPAR) 42, 639-644.

Reigber, C., Lhr, H., Schwintzer, P., 2002. CHAMP mission status. Advances in Space Research : The Official Journal of the Committee on Space Research (COSPAR) 30, 129-134.

Rich, F.J., Sultan, P.J., Burke, W.J., 2003. The 27-day variations of plasma densities and temperatures in the topside ionosphere. Journal of Geophysical Research 108, 1297.

Richards, P.G., 2001. Seasonal and solar cycle variations of the ionospheric peak electron density: Comparison of measurement and models. Journal of Geophysical Research 106, 12803-12819.

Stening, R.J., 1992. Modeling of the low latitude F region. Journal of Atmospheric and Terrestrial Physics 54, 1387-1412.

Tapley, B.D., Bettadpur, S., Ries, J.C., Thompson, P.F., Watkins, M.M., 2004. GRACE Measurements of Mass Variability in the Earth System. Science (New York, NY) 305, 503-505.

Tapping, K., Charrois, D., 1994. Limits to the accuracy of the $10.7 \mathrm{~cm}$ flux. Solar Physics 150, 305-315.

Thayer, J.P., Lei, J., Forbes, J.M., Sutton, E.K., Nerem, R.S., 2008. Thermospheric density oscillations due to periodic solar wind high-speed streams. Journal of Geophysical Research 113, A06307. 